PROCEEDINGS OF THE

AMERICAN MATHEMATICAL SOCIETY

Volume 129, Number 6, Pages 1727-1731

S 0002-9939(00)05701-4

Article electronically published on October 31, 2000

\title{
PRODUCTS OF EP OPERATORS ON HILBERT SPACES
}

\author{
DRAGAN S. DJORDJEVIĆ
}

(Communicated by Joseph A. Ball)

\begin{abstract}
A Hilbert space operator $A$ is called the EP operator if the range of $A$ is equal to the range of its adjoint $A^{*}$. In this article necessary and sufficient conditions are given for a product of two EP operators with closed ranges to be an EP operator with a closed range. Thus, a generalization of the well-known result of Hartwig and Katz (Linear Algebra Appl. 252 (1997), $339-345)$ is given.
\end{abstract}

\section{INTRODUCTION AND PRELIMINARIES}

Let $H, H_{1}, H_{2}$ be arbitrary Hilbert spaces, $\mathcal{L}\left(H_{1}, H_{2}\right)$ the set of all bounded operators from $H_{1}$ into $H_{2}$ and $\mathcal{L}(H, H)=\mathcal{L}(H)$. For $A \in \mathcal{L}\left(H_{1}, H_{2}\right)$ we use $A^{*}$, $\mathcal{R}(A)$ and $\mathcal{N}(A)$, respectively, to denote the adjoint operator, range and kernel of $A$.

If $\mathcal{R}(A)$ is closed, then $A^{\dagger} \in \mathcal{L}\left(H_{2}, H_{1}\right)$ is defined as the unique operator in $\mathcal{L}\left(H_{2}, H_{1}\right)$ which satisfies the equations:

$$
A A^{\dagger} A=A, \quad A^{\dagger} A A^{\dagger}=A^{\dagger}, \quad\left(A A^{\dagger}\right)^{*}=A A^{\dagger}, \quad\left(A^{\dagger} A\right)^{*}=A^{\dagger} A .
$$

$A^{\dagger}$ is known as the Moore-Penrose inverse of $A$ [2], 4].

For $A \in \mathcal{L}(H)$ consider the following inclusions: $\{0\} \subset \mathcal{N}(A) \subset \mathcal{N}\left(A^{2}\right) \subset \cdots$ and $H \supset \mathcal{R}(A) \supset \mathcal{R}\left(A^{2}\right) \supset \cdots$. The ascent of $A$ is defined as the least $k$ (if it exists) for which the following holds: $\mathcal{N}\left(A^{k}\right)=\mathcal{N}\left(A^{k+1}\right)$. If such $k$ does not exist, we say that the ascent of $A$ is equal to infinity. The descent of $A$ is defined as the least $k$ (if it exists) for which the following is satisfied: $\mathcal{R}\left(A^{k}\right)=\mathcal{R}\left(A^{k+1}\right)$. If such $k$ does not exist, we say that the descent of $A$ is equal to infinity. If the ascent and the descent of $A$ are finite, then they are equal, and this common value is known as the Drazin index of $A$, denoted by $\operatorname{ind}(A)$. If $\operatorname{ind}(A)=k$, then there exists the unique operator $A^{D} \in \mathcal{L}(H)$, which satisfies

$$
A^{k+1} A^{D}=A^{k}, \quad A^{D} A A^{D}=A^{D}, \quad A A^{D}=A^{D} A .
$$

$A^{D}$ is known as the Drazin inverse of $A$. If $\operatorname{ind}(A)=0$, then $A$ is invertible. If $\operatorname{ind}(A) \leq 1$, then $A^{D}$ is known as the group inverse of $A$, denoted by $A^{\#}$ [2, [4].

In this article we shall consider operators which satisfy $A^{D}=A^{\dagger}$. Notice that the last condition implies $\operatorname{ind}(A) \leq 1$; hence we shall consider operators which satisfy $A^{\#}=A^{\dagger}$.

Received by the editors May 4, 1999 and, in revised form, September 17, 1999.

2000 Mathematics Subject Classification. Primary 47A05, 15A09.

Key words and phrases. EP operators, generalized inverses.

(C)2000 American Mathematical Society 
Recall that an operator $A \in \mathcal{L}(H)$ is called the EP operator if $\mathcal{R}(A)=\mathcal{R}\left(A^{*}\right)$ [1], [5], [6].

If $A \in \mathcal{L}(H)$ has a closed range, then it is easy to see that $A$ is an EP operator if and only if $A^{\dagger}=A^{\#}$. Also, if $A$ is an EP operator, then $\mathcal{N}(A)=\mathcal{N}\left(A^{*}\right)$.

If $A$ and $B$ are two EP operators, it is a question when $A B$ is the EP operator. This problem for complex square matrices was open for twenty-five years (see [1, [5]). In [5] Hartwig and Katz found necessary and sufficient conditions for a product of two square EP matrices to also be the EP matrix. Their method cannot be applied to arbitrary Hilbert space operators.

In this paper we give necessary and sufficient conditions for a product of two EP operators with closed ranges to be the EP operator with a closed range. Thus, using a different method, we generalize the result from [5].

We shall mention the matrix decompositions of $A^{\dagger}$ and $A^{\#}$. If $M$ and $N$ are subspaces of $H$, then $M \dot{+} N$ denotes the direct (not necessary orthogonal) sum of $M$ and $N$. However, $M \oplus N$ denotes the orthogonal sum of $M$ and $N$.

If $A \in \mathcal{L}(H)$ has the group inverse, then $H=\mathcal{R}(A) \dot{+} \mathcal{N}(A)$ and $A$ has the following matrix decomposition:

$$
A=\left[\begin{array}{cc}
A_{1} & 0 \\
0 & 0
\end{array}\right]:\left[\begin{array}{l}
\mathcal{R}(A) \\
\mathcal{N}(A)
\end{array}\right] \rightarrow\left[\begin{array}{c}
\mathcal{R}(A) \\
\mathcal{N}(A)
\end{array}\right]
$$

where $A_{1}=\left.A\right|_{\mathcal{R}(A)}: \mathcal{R}(A) \rightarrow \mathcal{R}(A)$ is invertible. In this case the group inverse of $A$ has the form

$$
A^{\#}=\left[\begin{array}{cc}
A_{1}^{-1} & 0 \\
0 & 0
\end{array}\right] .
$$

If $A \in \mathcal{L}\left(H_{1}, H_{2}\right)$ and $\mathcal{R}(A)$ is closed, then $A$ has the following matrix decomposition with respect to the orthogonal sums of subspaces of $H_{1}$ and $H_{2}$ :

$$
A=\left[\begin{array}{cc}
\bar{A} & 0 \\
0 & 0
\end{array}\right]: H_{1}=\left[\begin{array}{c}
\mathcal{R}\left(A^{*}\right) \\
\mathcal{N}(A)
\end{array}\right] \rightarrow\left[\begin{array}{c}
\mathcal{R}(A) \\
\mathcal{N}\left(A^{*}\right)
\end{array}\right]=H_{2},
$$

where $\bar{A}=\left.A\right|_{\mathcal{R}\left(A^{*}\right)}: \mathcal{R}\left(A^{*}\right) \rightarrow \mathcal{R}(A)$ is invertible. The Moore-Penrose inverse of $A$ is given as

$$
A^{\dagger}=\left[\begin{array}{cc}
\bar{A}^{-1} & 0 \\
0 & 0
\end{array}\right]:\left[\begin{array}{c}
\mathcal{R}(A) \\
\mathcal{N}\left(A^{*}\right)
\end{array}\right] \rightarrow\left[\begin{array}{c}
\mathcal{R}\left(A^{*}\right) \\
\mathcal{N}(A)
\end{array}\right]
$$

If $A \in \mathcal{L}(H)$ is an EP operator with a closed range, then (1) becomes the decomposition of $A$ with respect to the orthogonal sum of subspaces.

\section{RESUlts}

Now, we state the main result of this paper.

Theorem 1. Let $A, B \in \mathcal{L}(H)$ be EP operators with closed ranges. Then the following statements are equivalent.

(a) $A B$ is an EP operator with a closed range.

(b) $\mathcal{R}(A B)=\mathcal{R}(A) \cap \mathcal{R}(B)$ and $\mathcal{N}(A B)=\mathcal{N}(A)+\mathcal{N}(B)$.

(c) $\mathcal{R}(A B)=\mathcal{R}(A) \cap \mathcal{R}(B)$ and $\mathcal{N}(A B)=\overline{\mathcal{N}(A)+\mathcal{N}(B)}$ 
Proof. (a) $\Longrightarrow(\mathrm{b})$ : Suppose that $A B$ is an EP operator with a closed range. Since $A$ is an EP operator, we get that the decomposition of $A$,

$$
A=\left[\begin{array}{cc}
A_{1} & 0 \\
0 & 0
\end{array}\right]:\left[\begin{array}{c}
\mathcal{R}(A) \\
\mathcal{N}(A)
\end{array}\right] \rightarrow\left[\begin{array}{c}
\mathcal{R}(A) \\
\mathcal{N}(A)
\end{array}\right],
$$

is a decomposition with respect to the orthogonal sum of subspaces. Since $B$ is an EP operator, it follows that $B$ has the following orthogonal decomposition:

$$
B=\left[\begin{array}{ll}
B_{1} & 0 \\
B_{2} & 0
\end{array}\right]:\left[\begin{array}{l}
\mathcal{R}(B) \\
\mathcal{N}(B)
\end{array}\right] \rightarrow\left[\begin{array}{l}
\mathcal{R}(A) \\
\mathcal{N}(A)
\end{array}\right] .
$$

We can write $\mathcal{R}(B)=\mathcal{N}\left(B_{1}\right) \oplus \mathcal{N}\left(B_{1}\right)^{\perp}$. It is easy to see $\mathcal{N}(A B)=\mathcal{N}(B) \oplus \mathcal{N}\left(B_{1}\right)$, so

$$
\mathcal{R}\left((A B)^{*}\right)=\mathcal{N}(A B)^{\perp}=\mathcal{N}\left(B_{1}\right)^{\perp} \subset \mathcal{R}(B) .
$$

Also, $\mathcal{R}(A B)=\mathcal{R}\left(A_{1} B_{1}\right)=A_{1}\left(\mathcal{R}\left(B_{1}\right)\right)$. Since $A B$ is an EP operator we get $\mathcal{N}\left(B_{1}\right)^{\perp}=\mathcal{R}(A B)$.

First, we prove $\mathcal{R}(A B)=\mathcal{R}(A) \cap \mathcal{R}(B)$. From $\mathcal{R}(A B)=\mathcal{N}\left(B_{1}\right)^{\perp} \subset \mathcal{R}(B)$ and $\mathcal{R}(A B) \subset \mathcal{R}(A)$, it follows that $\mathcal{N}\left(B_{1}\right)^{\perp}=\mathcal{R}(A B) \subset \mathcal{R}(A) \cap \mathcal{R}(B) \subset \mathcal{R}\left(B_{1}\right)$.

Let $Q$ be a projection from $H$ onto $\mathcal{R}(A)$ parallel to $\mathcal{N}(A)$ and let $P$ be a projection from $H$ onto $\mathcal{R}(B)$. By [4, Theorem 1, p. 127], we know that $\mathcal{R}(Q P)$ is closed if and only if $\mathcal{R}(A B)$ is closed. Hence, $\mathcal{R}(Q P)=Q(\mathcal{R}(B))=\mathcal{R}\left(B_{1}\right)$ is closed.

Suppose that there exists a closed subspace $S$ of $\mathcal{R}\left(B_{1}\right)$, such that

$$
\mathcal{N}\left(B_{1}\right)^{\perp} \oplus S=\mathcal{R}\left(B_{1}\right) .
$$

We conclude $S \subset \mathcal{N}\left(B_{1}\right) \oplus \mathcal{N}(B)$.

Define an operator $\overline{B_{1}} \in \mathcal{L}(H)$ in the following way: $\left.\overline{B_{1}}\right|_{\mathcal{R}(B)}=B_{1}$ and $\left.\overline{B_{1}}\right|_{\mathcal{N}(B)}$ $=0$. Then $\mathcal{R}\left(\overline{B_{1}}\right)=\mathcal{R}\left(B_{1}\right)$ and $\mathcal{N}\left(\overline{B_{1}}\right)=\mathcal{N}(B) \oplus \mathcal{N}\left(B_{1}\right)$.

Notice the following:

$$
\mathcal{R}\left({\overline{B_{1}}}^{2}\right)=\overline{B_{1}}\left(\mathcal{R}\left(B_{1}\right)\right)=B_{1}\left(\mathcal{N}\left(B_{1}\right)^{\perp}\right)=\mathcal{R}\left(B_{1}\right)=\mathcal{R}\left(\overline{B_{1}}\right)
$$

and

$$
\mathcal{N}\left({\overline{B_{1}}}^{2}\right)=\left[\mathcal{N}(B) \oplus \mathcal{N}\left(B_{1}\right)\right]+S=\mathcal{N}\left(\overline{B_{1}}\right) .
$$

It follows that the Drazin index of $\overline{B_{1}}$ is equal to zero or one; hence

$$
H=\mathcal{N}\left(\overline{B_{1}}\right)+\mathcal{R}\left(\overline{B_{1}}\right)=\left[\mathcal{N}(B) \oplus \mathcal{N}\left(B_{1}\right)\right]+\dot{R}\left(B_{1}\right)
$$

On the other hand, since $S \subset\left[\mathcal{N}(B) \oplus \mathcal{N}\left(B_{1}\right)\right] \cap \mathcal{R}\left(B_{1}\right)$ we conclude $S=\{0\}$. Finally, $\mathcal{N}\left(B_{1}\right)^{\perp}=\mathcal{R}(A) \cap \mathcal{R}(B)=\mathcal{R}\left(B_{1}\right)$ and $\mathcal{R}(A B)=\mathcal{R}(A) \cap \mathcal{R}(B)$.

To prove $\mathcal{N}(A B)=\mathcal{N}(A)+\mathcal{N}(B)$, consider the following decompositions:

$$
H=\mathcal{N}\left(B_{1}\right)^{\perp} \oplus \mathcal{N}\left(B_{1}\right) \oplus \mathcal{N}(B)=\mathcal{R}\left(B_{1}\right) \oplus \mathcal{R}\left(B_{1}\right)^{\perp} \oplus \mathcal{N}(A) .
$$

We conclude

$$
\mathcal{N}(A B)=\mathcal{N}(B) \oplus \mathcal{N}\left(B_{1}\right)=\mathcal{N}(A) \oplus \mathcal{R}\left(B_{1}\right)^{\perp}
$$

From $\mathcal{R}(B) \subset \mathcal{R}\left(B_{1}\right) \oplus \mathcal{R}\left(B_{2}\right)$ we get that $\mathcal{R}\left(B_{1}\right)^{\perp} \subset \mathcal{N}(B)$, and it follows $\mathcal{N}(A B)=\mathcal{N}(A)+\mathcal{N}(B)$.

(b) $\Longrightarrow(\mathrm{c})$ : This part of the proof is obvious. 
(c) $\Longrightarrow$ (a): Suppose that $\mathcal{R}(A B)=\mathcal{R}(A) \cap \mathcal{R}(B)$ and $\mathcal{N}(A B)=\overline{\mathcal{N}(A)+\mathcal{N}(B)}$. Immediately we get that $\mathcal{R}(A B)$ is closed. If $M$ and $N$ are subspaces of $H$, then $(M \cap N)^{\perp}=\overline{M^{\perp}+N^{\perp}}$. Now we find

$$
\begin{aligned}
\mathcal{R}(A B) & =(\mathcal{R}(A) \cap \mathcal{R}(B))^{\perp \perp}=\overline{\left(\overline{\left(\mathcal{R}(A)^{\perp}+\mathcal{R}(B)^{\perp}\right)}\right.} \\
& =(\overline{\mathcal{N}(A)+\mathcal{N}(B)})^{\perp}=\mathcal{N}(A B)^{\perp}=\mathcal{R}(A B)^{*}
\end{aligned}
$$

and finally, $A B$ is an EP operator with a closed range.

Our Theorem 1 is a generalization of the main result of Hartwig and Katz [5].

Remark 2. The statements (b) and (c) of Theorem 1 illustrate the difference between the finite and infinite dimensional Hilbert spaces. If $H$ is a finite dimensional Hilbert space, then the statement (b) is the same as the statement (c) of Theorem 1. In general Hilbert spaces, the statement (b) is a stronger condition than the statement (c) of Theorem 1.

It is well-known that the product of two selfadjoint operators $A, B$ is a selfadjoint operator if and only if $A B=B A$. In [6] it is shown that the product of two commuting EP matrices is again the EP matrix. For EP operators on an arbitrary Hilbert space the following result holds.

Theorem 3. If $A, B \in \mathcal{L}(H)$ are $E P$ operators with closed ranges and $A B=B A$, then $A B$ is the EP operator with a closed range also.

Proof. It is well-known that the group inverse $A^{\#}$ of $A$ commutes with every operator that commutes with $A$ [4, so we conclude that operators $A, B, A^{\#}, B^{\#}$ mutually commute. Obviously, $(A B)^{\#}=B^{\#} A^{\#}=B^{\dagger} A^{\dagger}$. Notice also

$$
\begin{aligned}
\left(A B B^{\#} A^{\#}\right)^{*} & =\left(B B^{\#} A A^{\#}\right)^{*}=\left(B B^{\dagger} A A^{\dagger}\right)^{*}=\left(A A^{\dagger}\right)^{*}\left(B B^{\dagger}\right)^{*}=A A^{\dagger} B B^{\dagger} \\
& =A B B^{\#} A^{\#} .
\end{aligned}
$$

The equality $\left(B^{\#} A^{\#} A B\right)^{*}=B^{\#} A^{\#} A B$ can be proved analogously. We have just proved

$$
(A B)^{\#}=B^{\#} A^{\#}=B^{\dagger} A^{\dagger}=(A B)^{\dagger} ;
$$

hence $A B$ is an EP operator with a closed range.

We can construct two noncommuting EP operators whose product is also an EP operator. Let $P$ be any orthogonal projection and let $A$ be any invertible operator which satisfies $A(\mathcal{N}(P))=\mathcal{N}(P)$ and $A(\mathcal{R}(P)) \nsubseteq \mathcal{R}(P)$. Then $P=P^{\dagger}=P^{\#}$ and $A^{-1}=A^{\dagger}=A^{\#}$. Also,

$$
\mathcal{R}(P) \cap \mathcal{R}(A)=\mathcal{R}(P)=\mathcal{R}(P A), \quad \mathcal{N}(P)+\mathcal{N}(A)=\mathcal{N}(P)=\mathcal{N}(P A) .
$$

By Theorem 1 it follows that $P A$ is an EP operator. On the other hand, $A$ and $P$ commute if and only if the decomposition $H=\mathcal{R}(P) \oplus \mathcal{N}(P)$ reduces $A$, i.e. $A(\mathcal{R}(P)) \subset \mathcal{R}(P)$ and $A(\mathcal{N}(P)) \subset \mathcal{N}(P)$. According to our construction we get $A P \neq P A$.

For a concrete realization of the preceding situation, consider the following example. 
Example 4. Let us take $H=\mathbb{C}^{2}, P=\left[\begin{array}{ll}1 & 0 \\ 0 & 0\end{array}\right]$ and $A=\left[\begin{array}{ll}1 & 0 \\ 1 & 1\end{array}\right]$. Then $P=P^{\dagger}=$ $P^{\#}$ and $A^{-1}=A^{\dagger}=A^{\#}=\left[\begin{array}{cc}1 & 0 \\ -1 & 1\end{array}\right]$; hence $P$ and $A$ are EP operators. Notice

$$
P A=P \neq\left[\begin{array}{ll}
1 & 0 \\
1 & 0
\end{array}\right]=A P .
$$

Now, $P, A$ and $P A$ are EP operators, but $A P \neq P A$.

If $\mathcal{R}(A), \mathcal{R}(B)$ and $\mathcal{R}(A B)$ are closed, then the rule $(A B)^{\dagger}=B^{\dagger} A^{\dagger}$ is called the reverse order rule for the Moore-Penrose inverse (and it does not hold in general). In [3] it is shown that if $\mathcal{R}(A), \mathcal{R}(B)$ and $\mathcal{R}(A B)$ are closed, then the following statements are equivalent:

(a) $(A B)^{\dagger}=B^{\dagger} A^{\dagger}$;

(b) $\mathcal{R}\left(A^{*} A B\right) \subset \mathcal{R}(B)$ and $\mathcal{R}\left(B B^{*} A^{*}\right) \subset \mathcal{R}\left(A^{*}\right)$.

In 1 Baskett and Katz considered the connection between the EP matrices and the reverse order rule for the Moore-Penrose inverse of matrices. To illustrate this connection, we formulate the following result.

Theorem 5. If $A, B \in \mathcal{L}(H)$ are EP operators with closed ranges and $\mathcal{R}(A)=$ $\mathcal{R}(B)$, then $(A B)^{\dagger}=B^{\dagger} A^{\dagger}$.

Proof. From $\mathcal{R}\left(A^{*}\right)=\mathcal{R}(A)=\mathcal{R}(B)=\mathcal{R}\left(B^{*}\right)$ we get

$$
\mathcal{R}\left(A^{*} A B\right)=A^{*} A\left(\mathcal{R}\left(A^{*}\right)\right)=A^{*}(\mathcal{R}(A))=\mathcal{R}\left(A^{*}\right)=\mathcal{R}(B)
$$

and

$$
\mathcal{R}\left(B B^{*} A^{*}\right)=B B^{*}(\mathcal{R}(B))=\mathcal{R}(B)=\mathcal{R}\left(A^{*}\right) .
$$

Hence, the reverse order rule $(A B)^{\dagger}=B^{\dagger} A^{\dagger}$ holds.

Some other similar and interesting results can be found in [1].

\section{ACKNOWLEDGEMENT}

I am grateful to the referee for helpful comments and suggestions concerning the paper.

\section{REFERENCES}

[1] T. S. Baskett and I. J. Katz, Theorems on products of EPr matrices, Linear Algebra Appl. 2 (1969), 87-103. MR 40:4280

[2] A. Ben-Israel and T. N. E. Greville, Generalized inverses: theory and applications, WileyInterscience, New York, 1974. MR 53:469

[3] R. H. Bouldin, Generalized inverses and factorizations, Recent applications of generalized inverses, Pitman Ser. Res. Notes in Math., vol. 66, 1982, pp. 233-249. MR 83j:47001

[4] S. R. Caradus, Generalized inverses and operator theory, Queen's paper in pure and applied mathematics, Queen's University, Kingston, Ontario, 1978. MR 81m:47003

[5] R. E. Hartwig and I. J. Katz, On products of EP matrices, Linear Algebra Appl. 252 (1997), 339-345. MR 98a:15050

[6] I. J. Katz, Weigmann type theorems for EP $P_{r}$ matrices, Duke Math. J. 32 (1965), 423-427. MR 31:4804

Department of Mathematics, Faculty of Philosophy, University of Niš, Ćirila i Metodija 2, 18000 Niš, Yugoslavia

E-mail address: dragan@archimed.filfak.ni.ac.yu

E-mail address: dragan@filfak.filfak.ni.ac.yu 